\title{
A Low-Cost Open-Hardware Wideband Multiple-Input-Multiple- Output (MIMO) Wireless Channel Sounder
}

Michael A. Jensen

jensen@byu.edu

Jon W. Wallace

wall@ieee.org

B. T. Maharaj

Louis P. Linde

Follow this and additional works at: https://scholarsarchive.byu.edu/facpub

Part of the Electrical and Computer Engineering Commons

\section{Original Publication Citation}

Maharaj, B. T., et al. "A Low-Cost Open-Hardware Wideband Multiple-Inputâ€"Multiple-Output (MIMO) Wireless Channel Sounder." Instrumentation and Measurement, IEEE Transactions on 57.1 (28): 2283-9

\section{BYU ScholarsArchive Citation}

Jensen, Michael A.; Wallace, Jon W.; Maharaj, B. T.; and Linde, Louis P., "A Low-Cost Open-Hardware Wideband Multiple-Input-Multiple-Output (MIMO) Wireless Channel Sounder" (2008). Faculty Publications. 160.

https://scholarsarchive.byu.edu/facpub/160

This Peer-Reviewed Article is brought to you for free and open access by BYU ScholarsArchive. It has been accepted for inclusion in Faculty Publications by an authorized administrator of BYU ScholarsArchive. For more information, please contact ellen_amatangelo@byu.edu. 


\title{
A Low-Cost Open-Hardware Wideband Multiple-Input-Multiple-Output (MIMO) Wireless Channel Sounder
}

\author{
B. T. Maharaj, Member, IEEE, Jon W. Wallace, Member, IEEE, \\ Michael A. Jensen, Fellow, IEEE, and Louis P. Linde, Senior Member, IEEE
}

\begin{abstract}
Due to the complexity of multiple-input-multipleoutput (MIMO) wireless channels, direct measurement is the main viable option for accurate characterization. Details on a new low-cost wideband channel sounder are presented, which was mainly constructed from conventional instruments and components. This switched architecture system is similar to commercial channel sounders and has modest cost $(<$ US\$ 50000). The sounder operates in the 2- to 8-GHz range with up to $100 \mathrm{MHz}$ of instantaneous bandwidth and supports eight transmitters and receivers, which are sufficient to support the development and assessment of current and future MIMO wireless systems. In this "open-hardware" project, the hardware design and software components are openly available to other researchers interested in developing or enhancing the MIMO measurement capability. The actual systems built at the University of Pretoria, Pretoria, South Africa, and Brigham Young University, Provo, UT, are presented, as are some example studies.
\end{abstract}

Index Terms-Measurement, multiple-input-multiple-output (MIMO), wideband.

\section{INTRODUCTION}

$\mathbf{T}$ HEORETICAL and experimental investigations have demonstrated the ability of wireless systems with multiple transmit (TX) and receive (RX) antennas to dramatically increase the spectral efficiency in environments with high multipath. These multiple-input-multiple-output (MIMO) wireless systems exploit the multiple transmission directions (or modes) afforded by a rich multipath. The highest capacity gains are possible when the fading coefficients of the MIMO channel transfer matrix are Rayleigh independent identically distributed (i.i.d.), which is accomplished by having rich multipath with widely separated antennas. Real environments and systems tend to exhibit a much more complicated MIMO behavior, and such channels are only captured by direct measurement (see [1] and references therein).

Manuscript received October 11, 2007; revised February 11, 2008. This work was supported in part by the National Research Foundation (NRF) under GUN 2053857, in part by the National Science Foundation under Information Technology Grant CCR-0313056, and in part by the U.S. Army Research Office under Multi-University Research Initiative (MURI) Grant W911NF04-1-0224.

B. T. Maharaj and L. P. Linde are with the Department of Electrical, Electronic, and Computer Engineering, University of Pretoria, Pretoria 0002, South Africa (e-mail: sunil.maharaj@mp.ac.za; 1linde@ postino.up.ac.za).

J. W. Wallace is with the School of Engineering and Science, Jacobs University Bremen, 28759 Bremen, Germany (e-mail: wall@ieee.org).

M. A. Jensen is with the Department of Electrical and Computer Engineering, Brigham Young University, Provo, UT 84602 USA (e-mail: jensen@ee.byu.edu).

Digital Object Identifier 10.1109/TIM.2008.919943
Although the theoretical channel capacity may be insensitive to the exact structure of the channel, a realistic system may heavily depend on this structure. For example, the doubledirectional clustering observed in measured channels suggests the advantage of directional elements [2]. Space-time codes designed with an understanding of the channel structure can outperform codes designed with the i.i.d. assumption [3]. Furthermore, multiuser communication performance may depend more heavily on the channel structure than single-user situations [4]. Since a detailed understanding of the channel is required for optimal system design and accurate performance assessment, direct measurement of MIMO channels continues to be important.

To partially fill the gap between the high cost of commercial channel sounders and the significant development time required to develop an instrument in-house, we present a new lowcost wideband MIMO channel sounder (operationally similar to commercial sounders [5]) that can be constructed at moderate cost by leveraging conventional off-the-shelf (COTS) instruments and modules. Details on hardware design and software components are openly available to other researchers interested in developing or enhancing the MIMO measurement capability. We hope that this "open-hardware" approach will lead to more cooperation in the area of MIMO channel measurement and modeling and simplify sharing and comparison of results. As much detail as possible is included in this paper to allow the system to be designed and deployed. However, due to space limitations, the reader is referred to other sources [6] for detailed schematics, software components, etc.

\section{Operational OVERVIEW}

To support current and future MIMO studies, a system that supports eight TX and eight RX antennas, a center frequency from 2 to $8 \mathrm{GHz}$, and $100 \mathrm{MHz}$ of instantaneous bandwidth is considered. Additional goals of short development time (less than six months) and low cost (< US\$ 50000) resulted in the system depicted in Fig. 1. The popular switched-array technique is employed, where antenna elements are connected to the TX and RX via high-speed microwave switches. Although simultaneous true array architectures exist, these tend to be expensive, require longer development, and complicate maintenance and calibration.

At the TX, a radio-frequency (RF) module mixes the wideband signal from the arbitrary waveform generator (AWG) with the local oscillator (LO) to up-convert to the microwave carrier. 


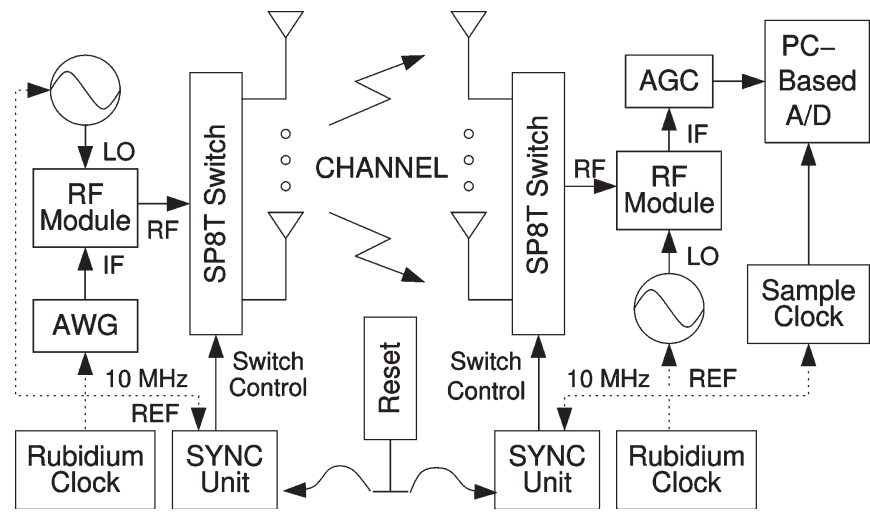

Fig. 1. High-level diagram of the wideband MIMO channel sounder.

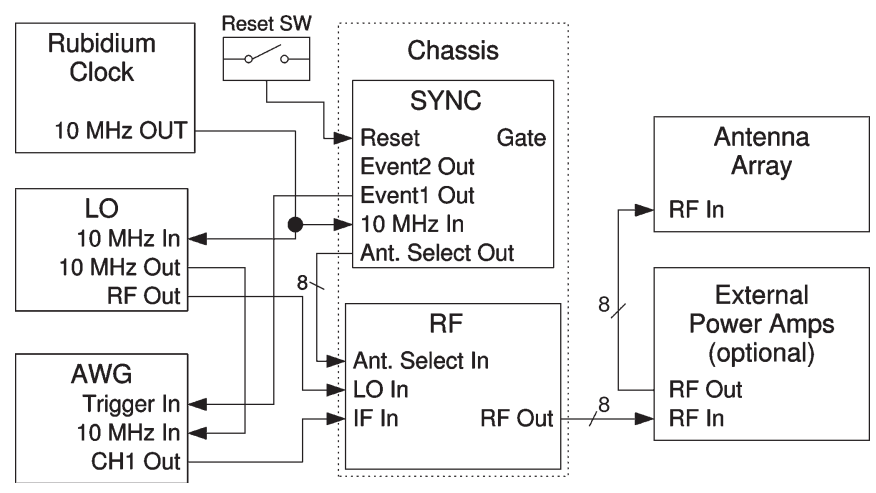

Fig. 2. Block diagram of the transmit subsystem.

This signal is power amplified and fed to a single-pole eightthrow (SP8T) switch, routing the signal to one of the eight TX antennas. At the RX, the signals on the antennas are routed via another SP8T switch to an RF module, consisting of lownoise amplification (LNA), single-stage down-conversion to an intermediate frequency (IF), and low-pass filtering (LPF). The IF signal is amplified by an automatic gain control (AGC) circuit and stored with a high-speed PC-based A/D card.

The coordinated switching of TX and RX arrays is accomplished by a custom-designed synchronization (SYNC) unit. The timing in the system is referenced to highly stable $10-\mathrm{MHz}$ rubidium time/frequency oscillators, ensuring proper timing and phase coherence.

\section{SySTEM COMPONENTS}

This section provides details on the specific hardware components shown in Fig. 1.

\section{A. Transmit Components}

Fig. 2 depicts a block diagram of the TX subsystem, which sequentially excites one of the $N_{T}$ antennas with a wideband signal. After generating a baseband probing signal on the AWG, the RF module up-converts this signal to the microwave carrier frequency by mixing with the LO. After amplification with a power amplifier (PA), a microwave switch routes the signal to one of the eight TX antenna elements, optionally followed by parallel PAs. The TX power level, with and without external PAs, is $5 \mathrm{~W}$ and $200 \mathrm{~mW}$, respectively, which is appropriate for outdoor and indoor measurements. Note that small to moderate

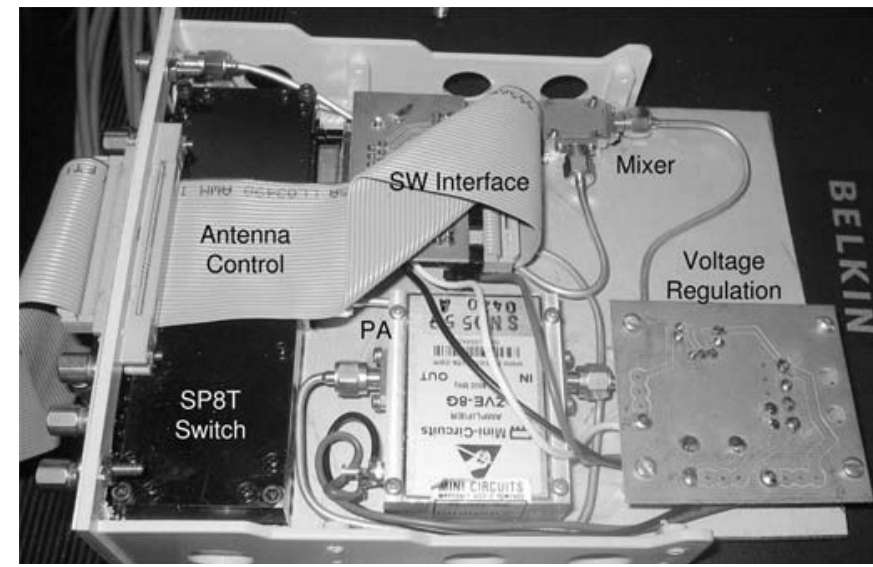

Fig. 3. Transmit RF module.

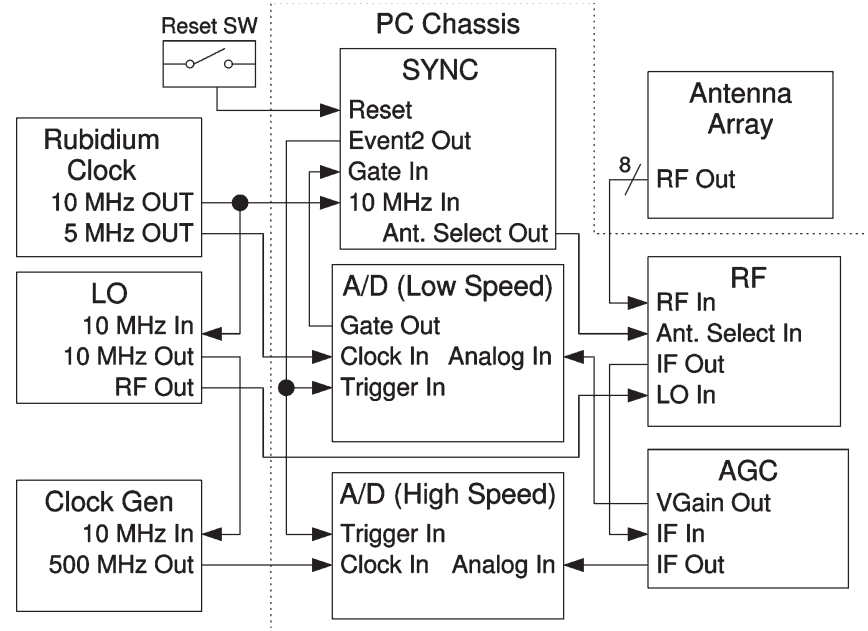

Fig. 4. Block diagram of the receive subsystem.

differences in the parallel PAs and switch branches are removed by the calibration procedure in Section III-F.

Fig. 3 depicts the TX RF module, consisting of COTS RF blocks connected via semi-rigid sub-miniature-A (SMA) cables, which is housed in a 5.25-in PC disk-drive frame and powered via $12 \mathrm{~V}$ to allow mounting inside a computer or disk-drive chassis. The SYNC unit (see Section III-C) supplies transistortransistor logic (TTL) enable lines to the microwave switches and an event trigger that releases each burst on the AWG.

\section{B. Receive Components}

Fig. 4 shows a block diagram of the RX subsystem, which samples the received wideband signal for all $N_{T} \times N_{R}$ antenna combinations. The RX RF module routes the signal from one of the $N_{R} \mathrm{RX}$ antennas to a broadband 40-dB LNA (NF = $2.5 \mathrm{~dB}$ ) and a mixer for down-conversion to a convenient IF. The IF signal is low-pass filtered, fed to the AGC module with $0-40 \mathrm{~dB}$ of gain, and sampled on a high-speed 8-bit 500-MS/s $\mathrm{A} / \mathrm{D}$ card. The VGain signal of the AGC is a power detector output whose voltage is proportional to the signal level in decibels. This signal is sampled at $1 \mathrm{MS} / \mathrm{s}$ with 16-bit resolution by an inexpensive low-speed multifunction input-output board. The maximum number of back-to-back snapshots is limited by the memory on the high-speed A/D card (512 MB) and 


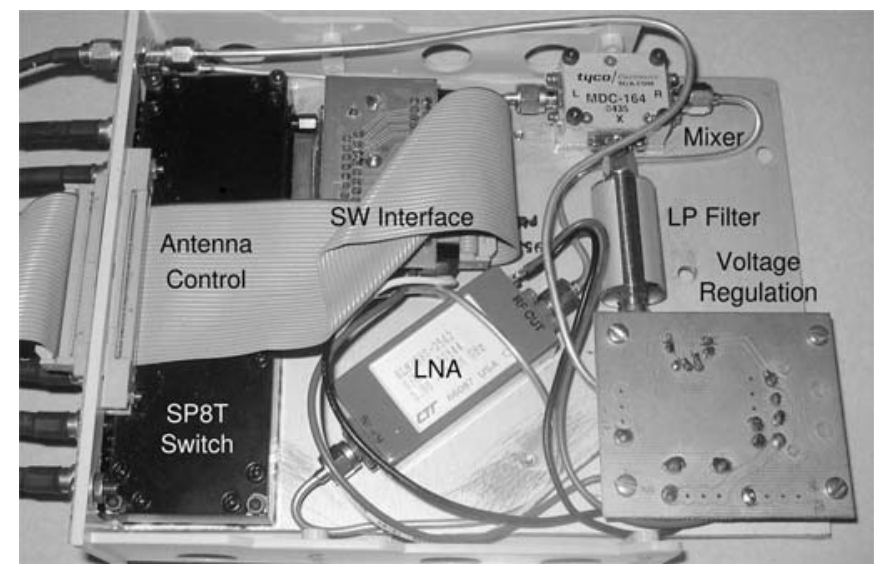

Fig. 5. Receive RF module.

the sample rate. For the maximum bandwidth, only $1 \mathrm{~s}$ of channels can be acquired before dumping to disk. For narrower bandwidths, the limitation is $1 \mathrm{~min}$, resulting from the lowspeed A/D board.

The RX switching rate is $1 / N_{T}$ times that of the TX subsystem to allow the channel from all $N_{T}$ transmitters to be measured for each of the $N_{R}$ receivers. The high- and lowspeed A/D boards (for measuring the wideband IF waveform and gain level, respectively) are simultaneously triggered by the Event2 signal that is asserted for each complete scan of the $N_{R}$ antennas. Simultaneous triggering of the two A/D cards is accomplished by the Gate signal that inhibits trigger events until both A/D cards are armed. Synchronized sample clocks are provided to both A/D cards (500 and $5 \mathrm{MHz}$ for high- and low-speed A/D, respectively).

Fig. 5 shows the interior of the RX RF module, again consisting of COTS RF blocks.

\section{Synchronization Module}

Key to the operation of switched-array MIMO sounding is coordinated switching at the TX and RX and triggering of TX bursts and RX acquisition windows. This is accomplished by a simple custom-designed SYNC unit, since no COTS solution was available.

Fig. 6 shows a simplified schematic of the SYNC unit, consisting of three 8-bit decrementing counters, where two counters form a 16-bit interval counter and the remaining 8 bits form an antenna counter. The interval counter controls the dwell time on each antenna, and this duration is set by rotary hex dials from 1 to $6553610-\mathrm{MHz}$ clocks (100 ns to $6.55 \mathrm{~ms}$ ). The antenna counter controls how many antennas must be cycled through (1-256).

The SYNC unit also provides critical event timing pulses. The Event1 signal, which is asserted when the interval counter reaches 0 , triggers the transmission of the wideband signal for each pair of selected antennas. The Event2 signal, which is asserted when the antenna counter reaches 0, triggers the RX acquisition of data at the beginning of each switching cycle.

The timing between the separate TX and RX SYNC units is synchronized by clocking each unit with a $10-\mathrm{MHz}$ rubidium oscillator. A simple trigger switch simultaneously connected to the two SYNC modules initially resets both units at the begin- ning of a measurement campaign. With well-tuned rubidium oscillators, the relative drift of timing events at TX to RX is approximately $50-100 \mathrm{~ns} / \mathrm{h}$.

\section{AGC Module}

In many communications environments, a large change in the RX power level with movement or time is possible due to fading and shadowing, and measurement sensitivity is enhanced using an AGC circuit. For this system, a simple AD8367-based circuit provides $0-40 \mathrm{~dB}$ of IF gain. The time constant of the AGC loop is approximately $5 \mu \mathrm{s}$, so that the gain can adapt for each new TX/RX pair. The low-speed A/D board samples the AGC loop output voltage, from which the gain can be computed and used to recover the true waveform from the recorded data.

\section{E. Wideband Probing Signal}

To enable the assessment of the channel response over a broad frequency range, the system uses a multitone excitation signal, where the number and spacing of tones can be controlled. The baseband multitone signal is of the form

$$
x(t)=\sum_{i=0}^{N_{F}-1} \cos \left(2 \pi f_{i} t+\phi_{i}\right)
$$

where $f_{i}$ and $\phi_{i}$ are the frequency and phase of the $i$ th tone, respectively. For equally spaced tones, $f_{i}=(i+0.5) \Delta f$. Note that each term in (1) actually represents two tones, so that the probing signal produces $2 N_{F}$ tones with a total probing bandwidth of $\left(2 N_{F}-1\right) \Delta f$. Postprocessing is simplified if each tone corresponds to a single fast Fourier transform (FFT) bin, which is guaranteed when $\Delta f=2 k f_{s} / N$, where $f_{s}$ is the sample rate, $N$ is the number of samples in each record, and $k$ is an integer.

An advantage of multitone signaling is that the channel response is directly obtained by selecting the appropriate FFT bins. One drawback, however, is that the envelope is not constant, and care must be taken to ensure that the linearity of TX PAs and switches is sufficient to avoid corruption of the measurements. The signal peak-to-average ratio is minimized by adjusting the phase on each of the tones. Here, the phases were optimized by simply realizing a large set of signals, each with a unique randomly generated phase weighting on the tones, and retaining the signal with the lowest peak-to-average ratio.

Since most p-i-n diode switches are not designed for hot switching (high level present during a switch operation), and channel estimation is simpler if the acquired signals for different antenna combinations have no temporal overlap, the multitone signal is multiplied by a Gaussian window of the form

$$
w(t)= \begin{cases}e^{-\left(T_{1}-t\right)^{2} /\left(2 \sigma^{2}\right)}, & 0 \leq t<T_{1} \\ e^{-\left(T_{2}-t\right)^{2} /\left(2 \sigma^{2}\right)}, & T-T_{2} \leq t \leq T \\ 1, & \text { otherwise }\end{cases}
$$

where $T_{1}$ and $T_{2}$ are the limits of the window, and the standard deviation $\sigma$ controls the rise and fall times of the window. Typically, the guard time $T_{1}+T_{2}$ is chosen to be much larger than the channel delay spread. The guard time also allows the AGC to adapt between switch events. 


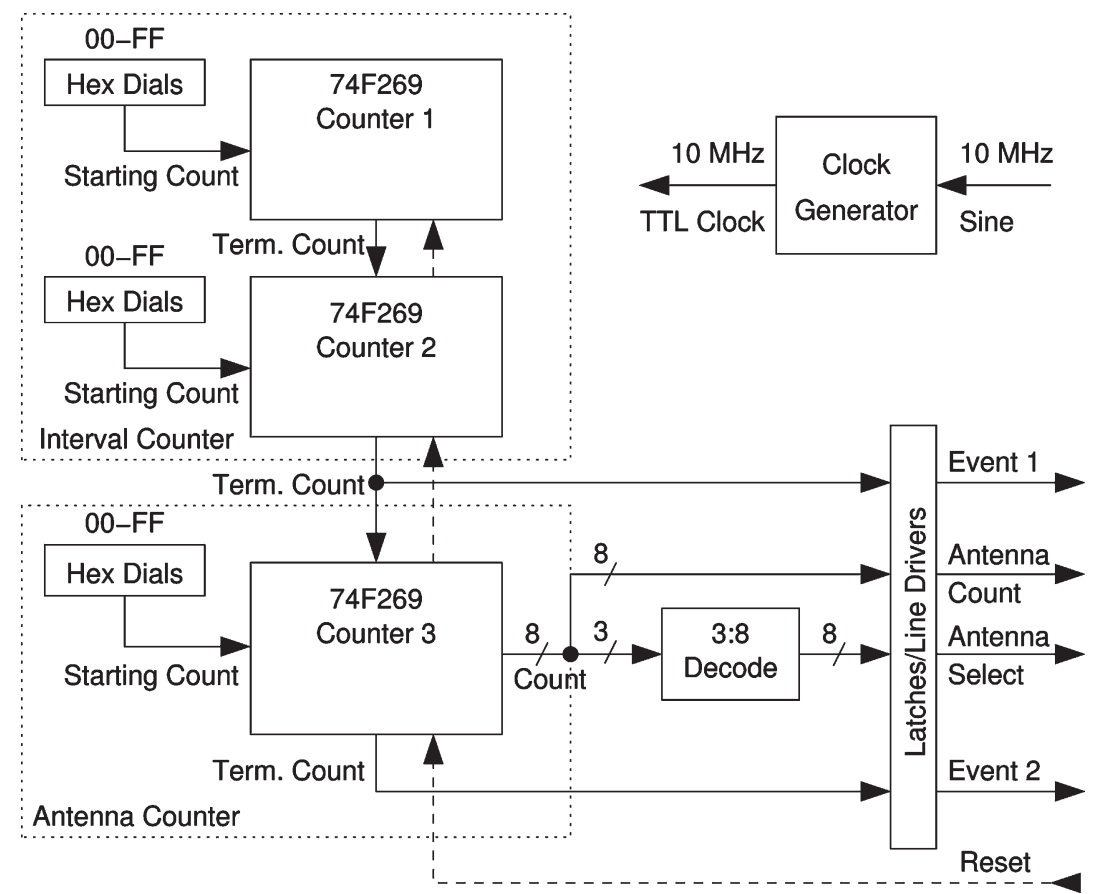

Fig. 6. Simplified schematic of the SYNC module.

\begin{tabular}{|l|ccccccc|}
\hline Parm. & $N_{F}$ & $\Delta f$ & $T_{1}$ & $T_{2}$ & $\sigma$ & $f_{s}$ & $N$ \\
\hline Value & 4 & $1 \mathrm{MHz}$ & $6 \mu \mathrm{s}$ & $7 \mu \mathrm{s}$ & $0.3 \mu \mathrm{s}$ & $400 \mathrm{MS} / \mathrm{s}$ & 20,000 \\
\hline
\end{tabular}
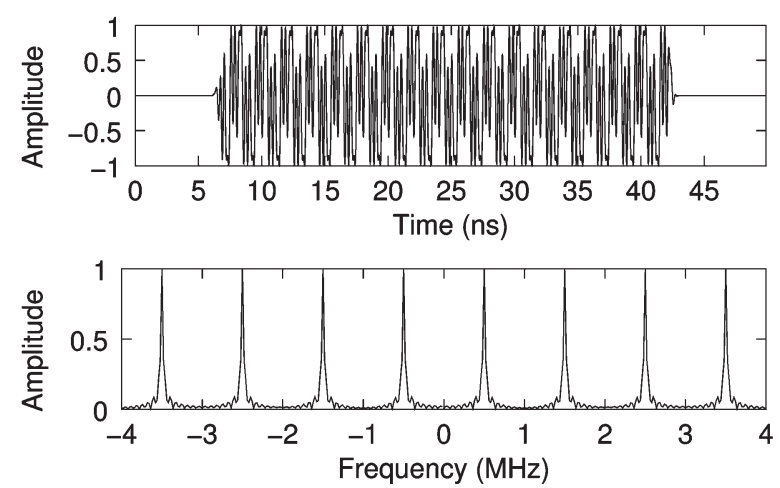

Fig. 7. Example multitone signal plotted versus time and frequency.

A simple 8-MHz multitone signal used for outdoor channel probing is depicted in Fig. 7. The window spreads the tones slightly, which is unavoidable for a finite-length signal. The active portion of the time-domain signal has a peak-to-average ratio of 1.74 , close to that of a sine wave $(\pi / 2 \approx 1.57)$.

\section{F. Calibration Procedure}

Since the RF components in the system are not ideal, raw measurements include the channel and system response. In addition, microwave switches are often constructed with unequal length transmission lines on various ports, leading to phase differences. These effects can be removed by a careful calibration method.

Fig. 8(a) depicts the measurement setup, where the ideal channel response from the $n$th TX antenna to the $m$ th RX antenna at baseband frequency $f_{k}$ is $h_{k, m n}$. One way to accomplish system calibration is to connect TX to RX, as depicted in (a)

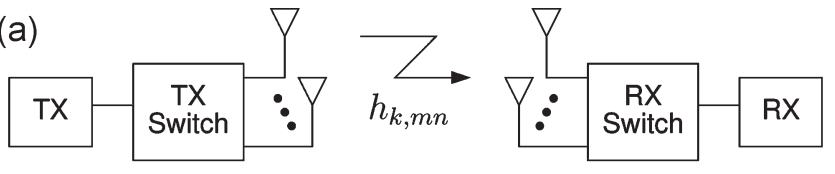

(b)

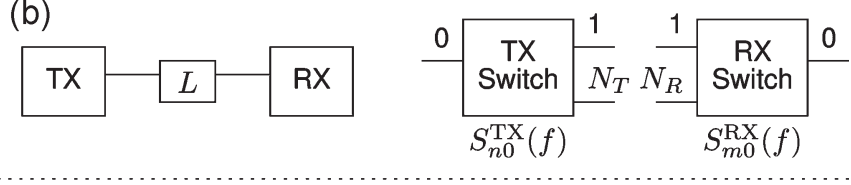

(c)

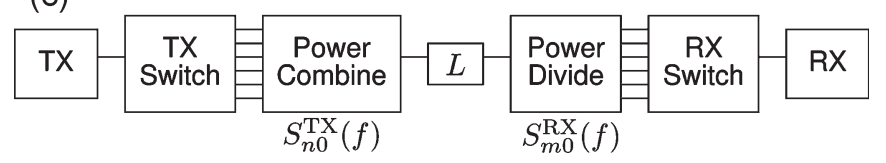

Fig. 8. Procedures for system calibration. (a) Original measurement setup. (b) Single-channel calibration. (c) Direct matrix calibration.

Fig. 8(b), where the switches and antennas have been replaced with a single attenuator with loss $L \mathrm{~W} / \mathrm{W}$. This measurement yields $y_{k}^{\text {cal }}$, which is the value of the FFT bin corresponding to the baseband tone at frequency $f_{k}$. The response of the switches is measured on a network analyzer giving broadband S-parameters $S_{n 0}^{\{\mathrm{TX}, \mathrm{RX}\}}(f)$, where 0 and $n$ are indices of common and switched ports, respectively, and $f$ is the frequency.

Assuming near-linear response of the RX subsystem, a measurement taken with the setup in Fig. 8(a) results in

$$
y_{k, m n}=L y_{k}^{\mathrm{cal}} h_{k, m n} S_{0 n}^{\mathrm{TX}}\left(f_{k}+f_{c}\right) S_{0 m}^{\mathrm{RX}}\left(f_{k}+f_{c}\right)
$$

where $k, m$, and $n$ are the frequency, $\mathrm{RX}$, and TX indices, and $f_{c}$ is the carrier frequency. The ideal (or calibrated) channel response $h$ is then obtained from

$$
h_{k, m n}=\frac{y_{k, m n}}{L y_{k}^{\mathrm{cal}} S_{0 n}^{\mathrm{TX}}\left(f_{k}+f_{c}\right) S_{0 m}^{\mathrm{RX}}\left(f_{k}+f_{c}\right)} .
$$



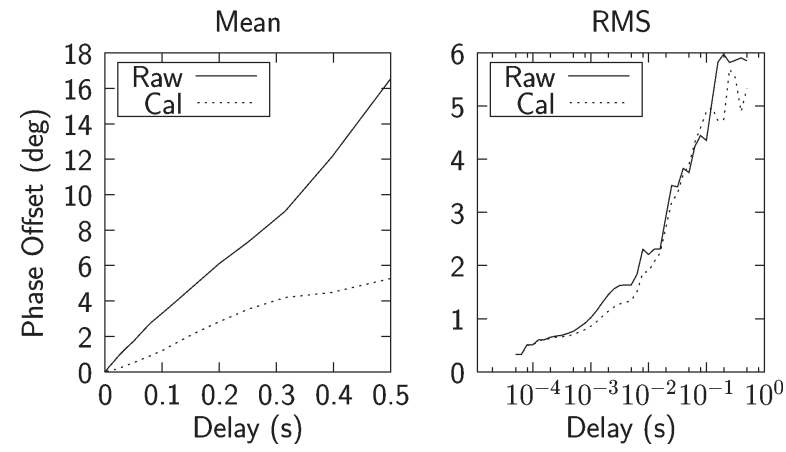

Fig. 9. Relative phase stability of LOs at $2.45 \mathrm{GHz}$.

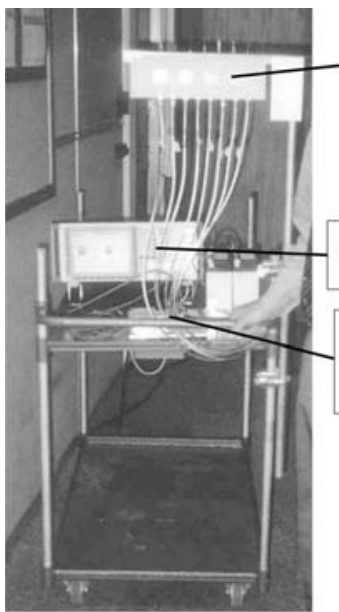

(a)

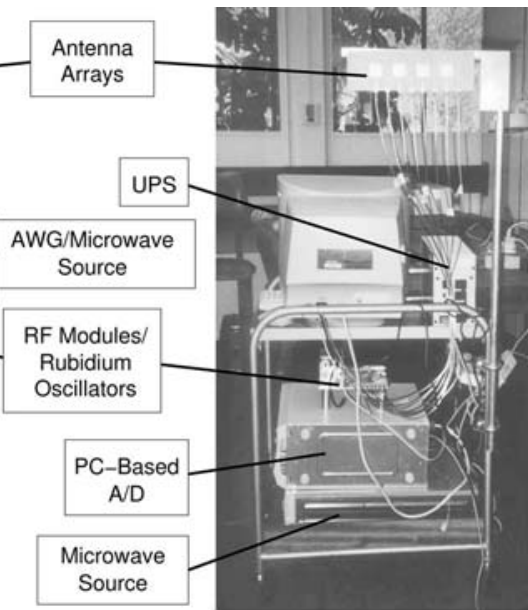

(b)
Fig. 10. System constructed and deployed at UP. (a) Transmit. (b) Receive.

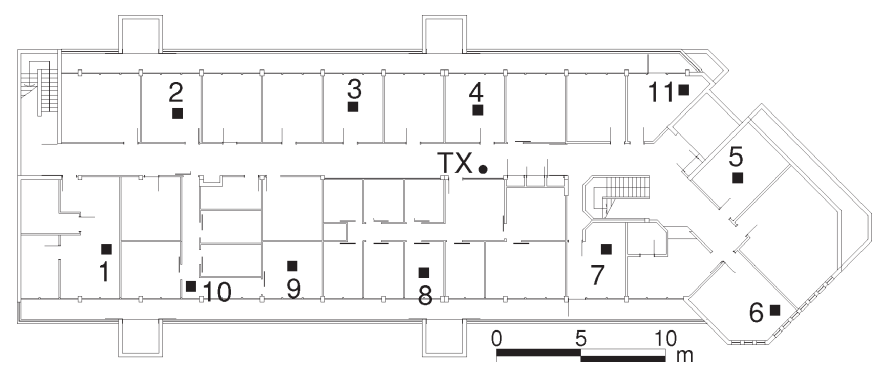

Fig. 11. Measurement scenario in the Carl and Emily Fuchs Engineering Building, UP

Another calibration method involves the connection strategy in Fig. 8(c). In this case, the switches remain, and TX and RX are connected via an $N_{T^{-}}$and $N_{R^{-} \text {way power combiner and }}$ divider, with measured S-parameters $S_{n 0}^{\{\mathrm{TX}, \mathrm{RX}\}}(f)$. To avoid RX saturation, a loss of $L$ may also be inserted. A measurement of all the TX and RX combinations yields $y_{k, m n}^{\mathrm{cal}}$. Similar to the first approach, the ideal channel response is computed as

$$
h_{k, m n}=\frac{y_{k, m n} S_{0 n}^{\mathrm{TX}}\left(f_{k}+f_{c}\right) S_{0 m}^{\mathrm{RX}}\left(f_{k}+f_{c}\right)}{L y_{k, m n}^{\mathrm{cal}}} .
$$

Note that the position of the S-parameters has changed since the divider and combiner are present in the calibration but not the measurement, whereas before, the switches were present in the measurement but not the calibration.

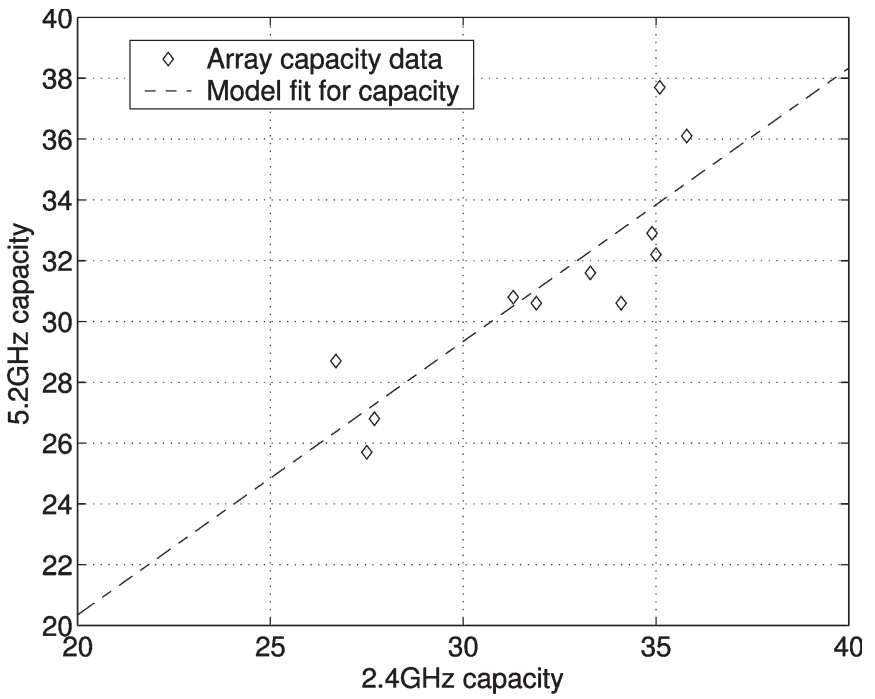

Fig. 12. Scatter plot of channel capacity at $5.2 \mathrm{GHz}$ versus that at $2.4 \mathrm{GHz}$, where each location is a data point.

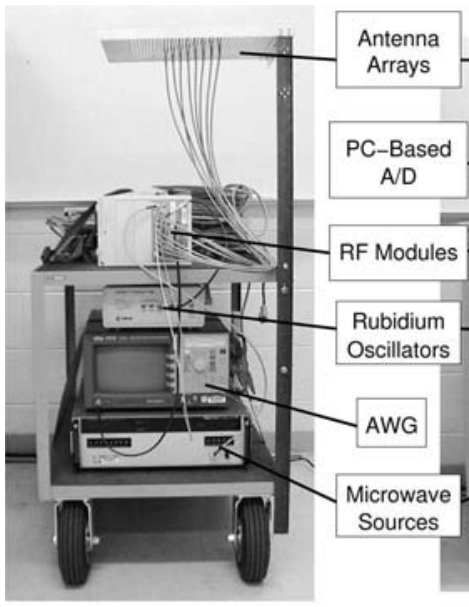

(a)

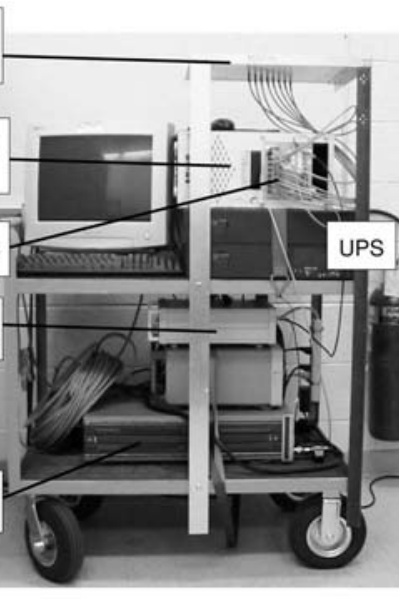

(b)
Fig. 13. Photos of the system constructed and deployed at BYU. (a) Transmit. (b) Receive.

\section{G. Phase Stability, Channel Stationarity, and Dynamic Range}

For accurate switched measurements, the acquisition time of an $N_{R} \times N_{T}$ channel measurement must be short relative to the time variability of the channel (Doppler) and phase drift of the LOs. With a dwell of $50 \mu \mathrm{s}$ and $N_{T}=N_{R}=8,3.2 \mathrm{~ms}$ is required for each acquisition, and a maximum Doppler of $156 \mathrm{~Hz}$ can be tolerated. Fig. 9 shows the mean and rms phase offset for a typical 1-s measurement with direct connection of TX to RX at $2.45 \mathrm{GHz}$. Raw curves indicate rubidium standards not calibrated for several months, and $\mathrm{Cal}$ indicate recent calibration. The deterministic mean phase drift (frequency offset) can be reduced by calibrating the standards, whereas the random phase fluctuations are caused by the phase noise of the LOs. The mean phase deviation for a 3.2-ms separation is well below $1^{\circ}$, and random fluctuations are approximately $2^{\circ} \mathrm{rms}$, which introduces negligible error for most MIMO studies.

Defining the RF dynamic range of the receiver as the span from the noise power level $(-110 \mathrm{dBm}$ at $1-\mathrm{MHz}$ bandwidth) to a power that is $3-\mathrm{dB}$ below amplifier saturation $(-40 \mathrm{dBm})$ 


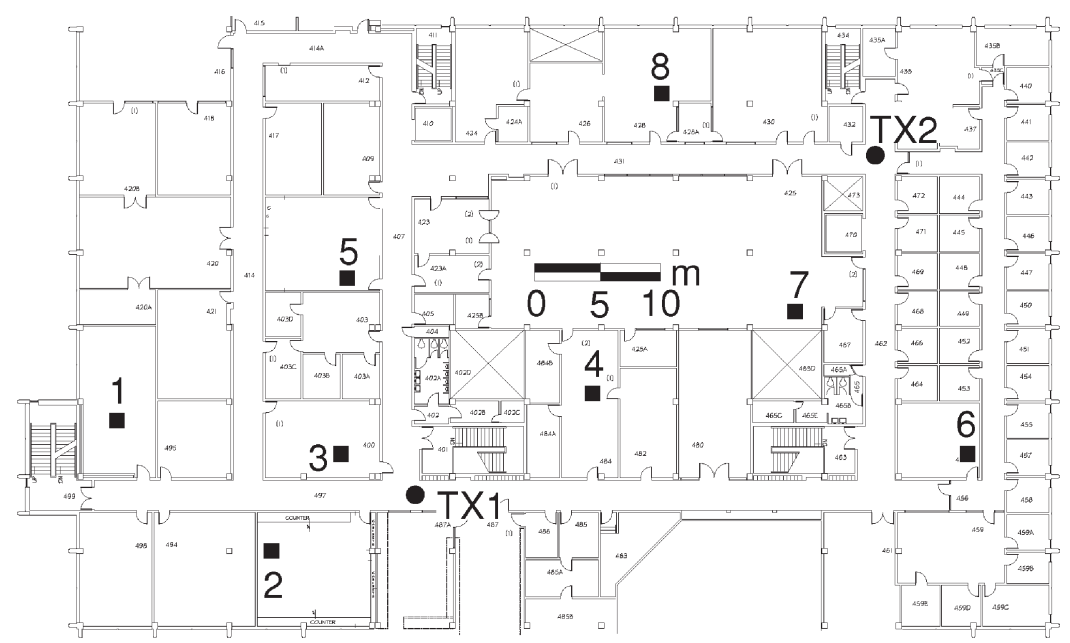

Fig. 14. Measurement scenario in the Clyde Building, BYU.

yields a value of $70 \mathrm{~dB}$. Because the system uses (and retains the gain level for) a 40-dB AGC circuit to amplify the RF signals to an appropriate level for the $\mathrm{A} / \mathrm{D}$, the system dynamic range between antennas (switch positions) and measurement snapshots is nearly the full RF dynamic range, with an achieved value of better than $60 \mathrm{~dB}$ in practice. Within a single measurement (switch position and snapshot), the dynamic range is mainly controlled by signal construction (tone orthogonality) and A/D quantization. For the signal depicted in Fig. 7, the dynamic range among frequency bins is better than $30 \mathrm{~dB}$. This can be enhanced by using a longer dwell time but at the expense of more phase variation.

\section{Example Systems And Measurements}

Nearly identical versions of the wideband MIMO channel sounder have been built and deployed at the University of Pretoria (UP), Pretoria, South Africa, and Brigham Young University (BYU), Provo, UT. This section describes these systems and briefly presents example results from each platform. For more details on these studies, see [7]-[9].

\section{A. UP System}

Fig. 10 depicts the UP system. The antenna arrays connected in the photo are dual-polarization patch antennas with $\lambda / 2$ interelement spacing. The TX consists of an integrated AWG/microwave source covering the $100-\mathrm{kHz}$ to $6-\mathrm{GHz}$ band. The rubidium oscillator module, RF components, and the SYNC unit are all powered via dedicated $12-\mathrm{V} / 24-\mathrm{V}$ rechargeable batteries. At the RX, an uninterruptible power supply (UPS) provides temporary backup power to the PC when the system must be unplugged for relocation. Again, the rubidium oscillator and RF module are powered via $12-\mathrm{V} / 24-\mathrm{V}$ batteries, whereas the SYNC module is inside the PC.

As an example study performed with this system, Fig. 11 shows a floor plan of the Carl and Emily Fuchs Electrical Engineering Building at UP, where colocated MIMO measurements were performed at 2.4 and $5.2 \mathrm{GHz}$. The TX and RX were placed in a central corridor and in several adjoining rooms, respectively. The antenna arrays were uniform linear arrays of monopoles with $\lambda / 2$ interelement spacing. One purpose of

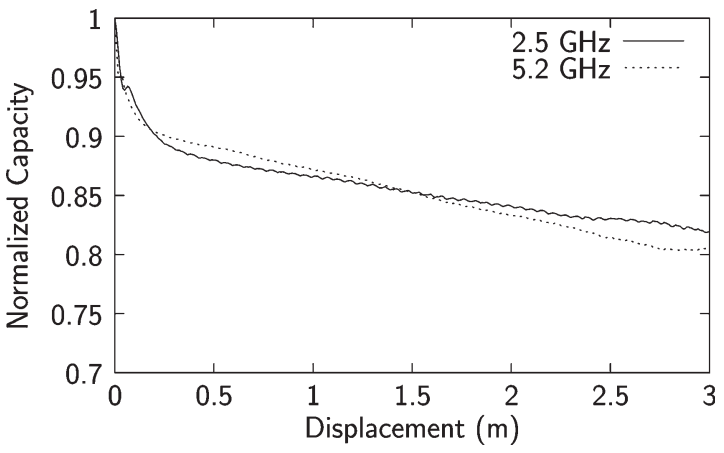

Fig. 15. Fractional reduction in capacity due to outdated transmit CSI with movement.

this experiment was to compare the effect of center frequency on element correlation and capacity. Fig. 12 plots the channel capacity at $5.2 \mathrm{GHz}$ versus that at $2.4 \mathrm{GHz}$, indicating a strong correlation in the channel quality at the two frequencies, and plots of TX and RX correlation also show similar dependence. These results indicate that the propagation mechanisms at the two frequencies may be similar, potentially saving effort in sitespecific channel characterization and network planning.

\section{B. BYU System}

Fig. 13 depicts the BYU system. The TX and RX arrays are monopole antennas mounted on a reconfigurable ground plane. At the TX, separate dedicated instruments are used for the rubidium oscillators, AWG, and microwave source. The RF module and SYNC unit are housed in a computer diskdrive chassis. At the RX, the PC houses the RF module, SYNC unit, and AGC board, and a high-capacity UPS is present for relocating the system.

Fig. 14 shows a map of the Clyde Building on the BYU campus, where measurements were performed at 2.5 and $5.2 \mathrm{GHz}$ as part of an effort to understand the effect of RX movement on the effective channel capacity. The TX was placed at one of two positions in the corridor. The antennas were eight-element uniform circular arrays of monopole elements separated by $\lambda / 2$. The RX was moved along a straight $4.5-\mathrm{m}$ path at the numbered boxes in various rooms. Fig. 15 depicts the reduction in channel 
capacity due to outdated TX channel state information (CSI) as a function of movement, averaged over all eight RX locations. The results indicate that TX CSI is useful for about $1 \mathrm{~m}$ of displacement, which is a longer distance than might be expected. In addition, the results at the two frequencies look most similar when plotted on a physical (not electrical) distance scale, suggesting that the effect is mainly due to shadow-type fading.

\section{CONClusion}

This paper has presented a low-cost wideband MIMO measurement platform constructed mainly from COTS instruments and components. This information should aid others in developing such measurement capabilities with reasonable time and cost, as well as encourage the sharing of MIMO data and the comparison of results.

\section{REFERENCES}

[1] M. A. Jensen and J. W. Wallace, "A review of antennas and propagation for MIMO wireless communications," IEEE Trans. Antennas Propag., vol. 52, no. 11, pp. 2810-2824, Nov. 2004.

[2] Q. H. Spencer, B. D. Jeffs, M. A. Jensen, and A. L. Swindlehurst, "Modeling the statistical time and angle of arrival characteristics of an indoor multipath channel," IEEE J. Sel. Areas Commun., vol. 18, no. 3, pp. 347360, Mar. 2000.

[3] B. Clerckx, C. Oestges, D. Vanhoenacker-Janvier, and L. Vandendorpe, "Robustness of space-time coding in spatially correlated fast-fading MIMO channels," in Proc. IEEE 62nd Veh. Technol. Conf., Dallas, TX, Sep. 25-28, 2005, vol. 4, pp. 2413-2417.

[4] Q. H. Spencer, C. B. Peel, A. L. Swindlehurst, and M. Haardt, "An introduction to the multi-user MIMO downlink," IEEE Commun. Mag., vol. 42, no. 10 , pp. 60-67, Oct. 2004.

[5] R. S. Thoma, D. Hampicke, A. Richter, G. Sommerkorn, A. Schneider, U. Trautwein, and W. Wirnitzer, "Identification of time-variant directional mobile radio channels," IEEE Trans. Instrum. Meas., vol. 49, no. 2, pp. 357-364, Apr. 2000.

[6] J. W. Wallace, "BYU wideband MIMO channel sounder technical reference manual," Dspace, Brigham Young Univ., Provo, UT, 2007. Tech. Rep. [Online]. Available: http://hdl.handle.net/1877/538

[7] B. T. Maharaj, J. W. Wallace, L. P. Linde, and M. A. Jensen, "Frequency scaling of spatial correlation from co-located 2.4 and $5.2 \mathrm{GHz}$ wideband indoor MIMO channel measurements," Electron. Lett., vol. 41, no. 6, pp. 336-337, Mar. 17, 2005.

[8] B. T. Maharaj, J. W. Wallace, L. P. Linde, and M. A. Jensen, "Linear dependence of double-directional spatial power spectra at 2.4 and $5.2 \mathrm{GHz}$ from indoor MIMO channel measurements," Electron. Lett., vol. 41, no. 24 , pp. 1338-1340, Nov. 24, 2005.

[9] J. Wallace and M. Jensen, "Time-varying MIMO channels: Measurement, analysis, and modeling," IEEE Trans. Antennas Propag., vol. 54, no. 11, pp. 3265-3273, Nov. 2006.

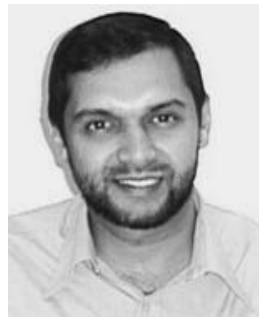

B. T. Maharaj (S'00-M'06) received the B.Sc.Eng. and M.Sc.Eng. degrees in electronic engineering from the University of Natal, Durban, Africa, and the M.Sc. degree in operational telecommunications (Merit) from the University of Coventry, Coventry, U.K., in 1996.

For about three years, he was a Microwave Design Engineer with the Electromagnetic Laboratory Pty Ltd. and Grinaker Avitronics Ltd. He was subsequently with Eastern Cape Technikon. He is currently the Acting Sentech Chair of broadband wireless communication (BWMC) with the Department of Electrical, Electronic, and Computer Engineering, University of Pretoria, Pretoria, South Africa.

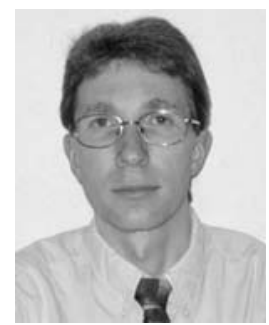

Jon W. Wallace (S'99-M'03) received the B.S. (summa cum laude) and Ph.D. degrees in electrical engineering from Brigham Young University (BYU), Provo, UT, in 1997 and 2002, respectively.

From 1995 to 1997, he was an Associate with Novell, Inc., Provo. In 1997, he was a member of technical staff with Lucent Technologies, Denver, CO. He received the National Science Foundation Graduate Fellowship in 1998 and was a Graduate Research Assistant with BYU until 2002. From 2002 to 2003, he was with the Mobile Communications Group, Vienna University of Technology, Vienna, Austria, where he was pursuing collaborative research in the area of wireless channel measurement and modeling. From 2003 to 2006, he was a Research Associate with the Wireless Communications Laboratory, BYU, developing platforms for wideband MIMO channel sounding and real-time, space-time coding. He is currently an Assistant Professor of electrical engineering with Jacobs University Bremen, Bremen, Germany. His current research interests include wireless channel sounding and modeling, MIMO communications, cognitive radio, and UWB and frequency-agile systems.

Dr. Wallace is serving as an Associate Editor of the IEEE TRANSACTIONS on ANTENNAS AND PROPAGATION.

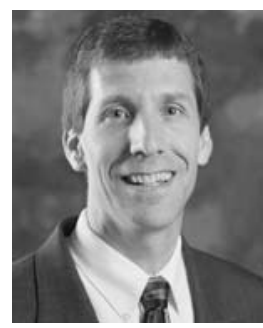

Michael A. Jensen (S'93-M'95-SM'01-F'08) received the B.S. (summa cum laude) and M.S. degrees in electrical engineering from Brigham Young University (BYU), Provo, UT, in 1990 and 1991, respectively, and the Ph.D. degree in electrical engineering from the University of California at Los Angeles (UCLA) in 1994.

From 1989 to 1991, he was a Graduate Research Assistant with the Lasers and Optics Laboratory, BYU. In 1990, he received a National Science Foundation Graduate Fellowship. From 1991 to 1994, he was a Graduate Student Researcher with the Antenna Laboratory, UCLA. Since 1994, he has been with the Department of Electrical and Computer Engineering, BYU, where he is currently a Professor and Department Chair. His main research interests include antennas and propagation for personal communications, radar remote sensing, numerical electromagnetics, and optical fiber communications.

Dr. Jensen is a member of Eta Kappa Nu and Tau Beta Pi. He currently chairs the Joint Meetings Committee for the IEEE Antennas and Propagation Society. He has been an Associate Editor for the IEEE TRANSACTIONS ON ANTENNAS AND PROPAGATION, a member of the Society Administrative Committee, and Co-chair and Technical Program Chair for several symposia. He received the H. A. Wheeler paper award of the IEEE TRANSACTIONS ON ANTENNAS AND Propagation in 2002 and the Best Student Paper Award at the 1994 IEEE International Symposium on Antennas and Propagation.

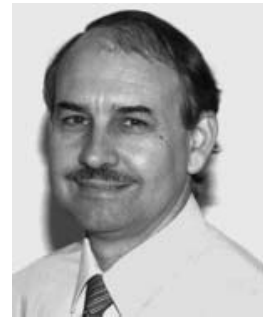

Louis P. Linde (M'80-SM'90) received the Hons. B.Eng. (electrotechnical) degree from the University of Stellenbosch, Stellenbosch, South Africa, in 1973 and the M.Eng. (Electronic) (cum laude) and D.Eng. degrees from the University of Pretoria, Pretoria, South Africa, in 1980 and 1983, respectively.

He is currently a Professor and the Group Head of the Signal Processing and Telecommunications Division with the Department of Electrical, Electronic, and Computer Engineering, University of Pretoria, where he is also the Director of the Centre for Radio and Digital Communication (CRDC). His research interests are in the fields of digital modulation and coding for fading channels, CDMA spreading sequence design, synchronization and equalization methods for highly mobile communication applications, and MIMO space-time coding.

Prof. Linde is the Specialist Editor for Telecommunications, Signal Processing, and Information Theory of the SAIEE Africa Research Journal. 